\title{
Ion density fluctuations in liquid metals: the strongly interacting ion-electron plasma
}

\author{
L.E.Bove ${ }^{1}$, C.Petrillo², F.Sacchetti ${ }^{2}$ \\ ${ }^{1}$ Department Physique des Milieux Denses CNRS-IMPMC, Universitè Paris 6, F-75015 Paris, France \\ 2 Dipartimento di Fisica, Università di Perugia, I-06123 Perugia, Italy and CNR-INFM CRS-Soft, Roma
}

Received November 20, 2007

\begin{abstract}
An unified description of liquid metals dynamics based on an interacting two-component model for the ionelectron plasma is tempted. The propagation velocity of the collective modes in alkali and polyvalent metals, derived from inelastic neutron and x-rays scattering experiments, is compared with the estimates obtained by different theoretical approximations for the strongly-interacting plasma. Using accurate results for the electron gas correlation energy, and taking into account the ion finite size effects, a good overall description of a large set of liquid metals is obtained. The observed trend for the damping of the collective modes in liquid metals is
\end{abstract} also discussed within the framework of the two-component description.

Key words: liquid metals, dynamics, inelastic neutron and $x$-rays scattering, electron density

PACS: $61.12 .-q, 61.25 . \mathrm{Mv}, 71.10 . \mathrm{Ca}$

Liquid metals are exceptional systems catalyzing the interest of different disciplines spreading from fundamental physics, to industrial chemistry and earth science. Broad industrial applications and a rather simple manufacturing make liquid metals materials of high technological relevance. Liquid metals also represent the paradigm of simple liquids: they embed the physical properties of real fluids without bringing in the additional complexity of orientational and internal vibrational degrees of freedom. These characteristics, as well as their handiness, make liquid metals ideal systems for the experimental investigation of the microscopic dynamics of liquids. In crystals, the vibrational dynamics of atoms is described by phonon propagation with damping mostly due to anharmonicity effects and electron-phonon interaction. In liquids, structural effects (the random distributions of particles) and dynamical effects (the diffusion and the relaxation processes) make the modelling of vibrational dynamics still more complex. Quite generally, density fluctuation excitations are observed in most liquids with a dispersion relation, i.e. a definite relationship between frequency $\omega(Q)$ and wavevector $Q$, which extends well beyond the hydrodynamic limit. What distinguishes liquid metals from other simple liquids is their peculiar ability to sustain welldefined propagating density fluctuations $[1-5]$ up to about $Q_{0}$, the position of the first peak in the static structure factor (see figure 1). Conversely, in uncharged simple liquids, like Lennard-Jones (LJ) liquids, the excitations appear to be strongly over-damped just above a small fractional value of $Q_{0}$ [6]. This different behaviour could be connected to the presence of a nearly free electron gas in liquid metals, which softens the short range part of the pair potential and favours a certain coherence of the ion-density fluctuations on local scale. The presence of a radius of correlation of the electron gas $r_{\mathrm{s}}=(9 \pi / 4)^{1 / 3} / k_{\mathrm{F}}$, introduces a pseudo-periodicity in the momentum space on a scale commensurable to the Fermi momentum $k_{\mathrm{F}}$, then roughly matching $Q_{0}$ [7]. Consequently, liquid metals show an intermediate character, with respect to the effects of structural disorder, between simple liquids and crystals having force-constants disorder (with an order parameter dictated by $k_{\mathrm{F}}$ ). Since the pioneering experiment of Copley and Rowe [1] on liquid Rubidium the peculiar dynamical behaviour of liquid metals has drawn attention to both unravelling the mechanism governing the propagation of density fluctuations in disordered systems, and to addressing the complex field of screening phenomena in ion plasma. For the last ten years, inelastic X-ray (IXS) and neutron (INS) scattering experiments, which are optimally suited to probing the dynamic 
response at $\mathrm{THz}$ frequencies and sub-nm scale, have provided a wide and consistent body of data on the dynamic structure factor in different classes of liquid metals [1-5,8-10]. It is now becoming commonly accepted that a quantitative and unified description of the dynamical behaviour of liquid metals cannot be obtained if the screened Coulomb interaction is not properly treated.
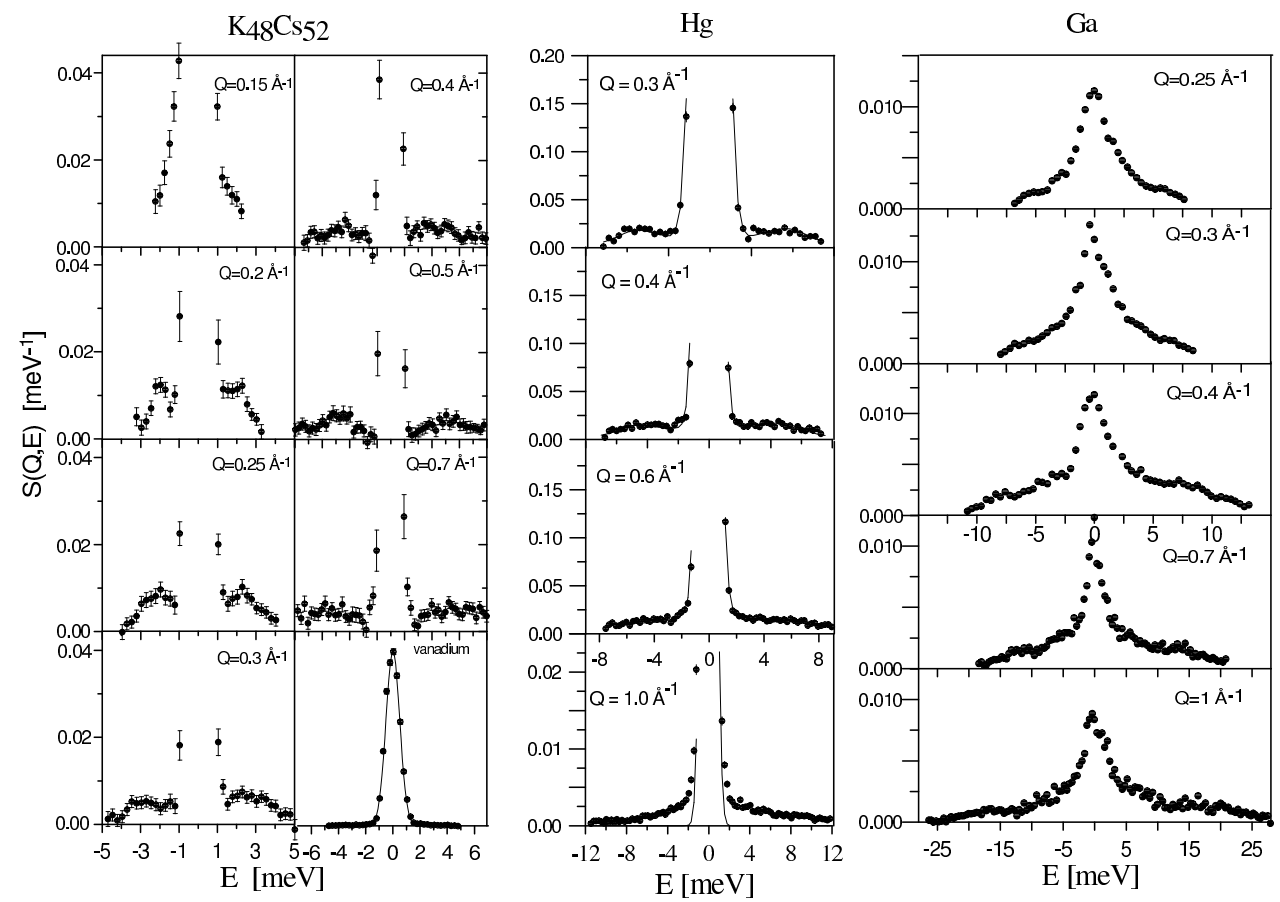

Figure 1. Some selected spectra of liquid metals in the mesoscopic region measured by INS.

We made a first attempt to describe the dynamics of liquid alkali metals on a common ground simply resorting to an electron density based scaling via the $r_{\mathrm{s}}$ parameter [3]. Ground for this simplified approach was provided by the results of an experimental study on the dynamics of an alkali metal liquid binary alloy, namely $\mathrm{K}_{52} \mathrm{Cs}_{48}$, with a composition matching the $r_{\mathrm{s}}$ values of pure $\mathrm{Rb}$. A scaling law of the dispersion relations associated to the ion density fluctuations of the two systems was obtained by only taking into account the effective mass of the alloy. This emphasizes the key role of the electron density against the ion-core dependent contributions which are mainly described by the ion plasma frequency. Indeed, even though the repulsive core is quite different in the two systems, the scaling of the dispersion curves can be obtained without resorting to it [3]. To check for possible extension of this scaling to the whole class of alkali metals, hence to systems with different electron densities and consequently different dielectric functions, we resorted to the simple Bohm-Staver model [11] to describe the density fluctuation propagation in the coupled electron-ion plasma. This model describes the ions as point-like particles moving in the homogeneous electron gas. In their motion, ions polarize the surrounding electron gas which almost instantaneously piles up around the ionic charges thus causing a renormalization of the force constants and hence of the frequencies associated to the density fluctuations. In this approximation, one has:

$$
\omega^{2}(Q)=\frac{\Omega_{\mathrm{p}}^{2}}{\epsilon(Q)},
$$

where the plasma frequency of the ionic gas is given by $\Omega_{\mathrm{p}}^{2}=4 \pi n(Z e)^{2} / M, n,(Z e)$ and $M$ being the number density, the charge and the mass of the ion. $\epsilon(Q)$ is the static dielectric function of the homogeneous electron gas at the appropriate density. Exploiting this simple model with the electron dielectric function calculated in the random phase approximation (RPA) [11], we estimated the renormalized frequencies of the longitudinal modes. A simple scaling law of the dispersion curves of 
heavy alkali metals ruled by the electron density was obtained, see figure 2, thus providing a clear evidence of the renormalization effects brought about by the electron gas on the ionic collective dynamics. The observed scaling also emphasizes that $\mathrm{K}, \mathrm{Rb}$, and $\mathrm{Cs}$ are described by a similar repulsive potential [4], whose effects on the dispersion curve are confined to a secondary role.

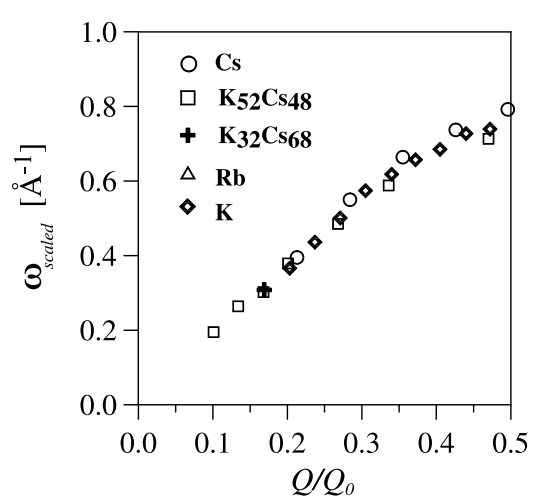

Figure 2. Dispersion relation of the collective excitations in $\mathrm{K}_{52} \mathrm{Cs}_{48}, \mathrm{~K}_{32} \mathrm{Cs}_{68}$, pure $\mathrm{K}$, pure $\mathrm{Rb}$ and pure Cs [1-4] as scaled according to: $\omega(Q)_{\text {scaled }}=\omega(Q) Q \sqrt{\frac{\epsilon(Q)}{\Omega_{\mathrm{p}}^{2}}}$ and versus $Q / Q_{0}$.



Figure 3. The measured propagation velocity $c_{\text {exp }}$ in alkali metals, as a function of $r_{\mathrm{s}}$, compared to the BS prediction in the RPA.

Applying this scheme we could also estimate the absolute values of the collective mode velocities, which are obtained by taking the long-wavelength limit of the electron dielectric function.

$$
c_{\mathrm{RPA}}(Q)=\frac{\omega(Q)}{Q}=\frac{1}{Q} \sqrt{\frac{\Omega_{\mathrm{p}}^{2}}{\epsilon(Q)}} \cong \frac{\Omega_{\mathrm{p}}}{k_{s}},
$$

where $k_{\mathrm{s}}$ is the exact screening wavevector, which coincides with the Thomas-Fermi wavevector $k_{\mathrm{TF}}$ in the RPA. Equation (2) provides a simple prescription for estimating a parameter directly measured by INS and IXS experiments. The results of INS and IXS experiments show that the values of sound velocity, $c_{\exp }$, measured in the $\mathrm{THz}$ regime are markedly larger than the values obtained by ultrasonic measurements in the adiabatic regime, $c_{0}$ [12]. The enhanced value of $c_{\exp }$ corresponds to the reply of the frozen system to the high frequency probe. The marked difference between $c_{\exp }$ and $c_{0}$ observed in some liquid metals, compared to uncharged simple liquids, can be ascribed to the short range modification of the ionic pair potential by the screening effects of the interacting electron gas [13]. A quantitative comparison of the measured high frequency sound velocity with the output of theoretical models could be fundamental in order to unravel the details of the potential which are significant for the collective dynamics. Despite the excellent quantitative agreement observed in single cases ( $\mathrm{Na}$, for example), the values of $c_{\exp }$ estimated from equation (2) for the series of molten alkalis provided unsatisfactory results [4] (see figure 3). This failure can be partially ascribed to the omission, in the RPA estimation of the dielectric function, of the effects related to the electron correlations, which become more and more important in going from Li to Cs. In fact, the RPA prescription gives reliable results in the high electron density regime, i.e. when $r_{\mathrm{s}}$ is small enough, where the kinetic energy dominates over the interaction term in a jellium model, but fails in describing the electron gas response in the low electron density regime, where the electron correlation effects play a major role [11]. However, as shown in figure 3, the deviations from the predicted RPA values are, as expected, positive and sizeable for potassium, rubidium and caesium which have a low electron density, whereas negative and large for liquid lithium, that is the system with the highest electron density. This indicates a failure, someway, of the simple BS scheme. Also the fact that this deviation does not show a simple increasing trend with $r_{\mathrm{s}}$ indicates that a description based on a single parameter, the electron density, is too simple an approach. Actually, it is also known that the RPA fails rather soon as a function of $r_{\mathrm{s}}$ in providing a correct value for $k_{\mathrm{s}}$. 
An improved description [4,14] of the electron gas response, which takes into account the electron correlation effects, can be obtained by introducing the local field effects in the dielectric function. The Thomas-Fermi wavevector in equation (2) is substituted by the exact screening wavevector $k_{\mathrm{s}}$ derived by the third moment sum rule:

$$
k_{\mathrm{s}}^{2}=\frac{k_{\mathrm{TF}}^{2}}{1-\lambda k_{\mathrm{TF}}^{2}}
$$

with the low- $Q$, zero-frequency, local field coefficient $\lambda$ given by [15]

$$
\lambda=\frac{1}{4 k_{\mathrm{F}}^{2}}\left(1+\frac{\pi}{3}(4 / 9 \pi)^{1 / 3} r_{\mathrm{s}}^{2} \frac{\partial \epsilon_{\mathrm{c}}}{\partial r_{\mathrm{s}}}-\frac{\pi}{6}(4 / 9 \pi)^{1 / 3} r_{\mathrm{s}}^{3} \frac{\partial^{2} \epsilon_{\mathrm{c}}}{\partial r_{\mathrm{s}}^{2}}\right) .
$$

Here $\epsilon_{\mathrm{c}}$ is the correlation energy per particle of the uniform interacting electron gas [11], which can be estimated by the interpolation formulas provided by quantum Monte Carlo simulations [16]. Exploiting the interpolation scheme described in [16], the compressibility of the homogeneous electron gas, and hence $k_{\mathrm{s}}^{2}$, becomes negative at $r_{\mathrm{s}}=5.45$, a value smaller than that corresponding to Cs. As a consequence, the collective modes of the point-like ions cannot propagate when $r_{\mathrm{s}}$ is greater than the above limiting value or even the system cannot be in a stable state. Considering that the stability of real systems, among which heavy alkali metals, is not affected by them attaining $r_{\mathrm{s}}$ values close to or even beyond this threshold, the effect of the ion finite size on the stability is crucial, especially for the low electron density systems. This is at the basis of the large, and in the case of Cs unphysical, discrepancy observed in heavy alkali metals when comparing the exact evaluation of the propagation velocity of density fluctuations with the corresponding measured values, as reported in table I.

Table 1. $c_{\mathrm{RPA}}$ is RPA estimate of the collective mode velocity, $c_{\mathrm{lf}}$ is the estimate deduced from equation (3), i.e. without including ionic core effects, and $c_{\exp }$ the experimental value. $\left\langle R^{2}\right\rangle / 6+D_{\mathrm{p}}$ is the ionic core contribution (see text) as described in equation (6).

\begin{tabular}{|c|c|c|c|c|c|}
\hline System & $r_{\mathrm{s}}$ & $c_{\mathrm{RPA}}$ & $c_{\text {lf }}$ & $c_{\text {exp }}$ & $\left\langle R^{2}\right\rangle / 6+D_{\mathrm{p}}$ \\
\hline & & $\mathrm{meV} \mathrm{\AA}$ & $\mathrm{meV}$ & $\mathrm{meV} \AA$ & $\AA^{2}$ \\
\hline $\mathrm{Li}$ & 3.250 & 43.931 & 28.577 & 36.450 & $0.099 \pm 0.042$ \\
$\mathrm{Na}$ & 4.014 & 19.155 & 10.115 & 18.500 & $0.301 \pm 0.046$ \\
$\mathrm{~K}$ & 4.889 & 12.420 & 4.131 & 15.500 & $0.811 \pm 0.113$ \\
$\mathrm{Rb}$ & 5.204 & 7.969 & 1.775 & 9.210 & $0.767 \pm 0.086$ \\
$\mathrm{~K}_{52} \mathrm{Cs}_{48}$ & 4.789 & 10.363 & 3.737 & 11.200 & $0.570 \pm 0.080$ \\
$\mathrm{Cs}$ & 5.586 & 6.036 & -0.957 & 7.460 & $0.962 \pm 0.105$ \\
$\mathrm{Al}$ & 2.191 & 52.142 & 41.005 & 40.000 & $-0.008 \pm 0.030$ \\
$\mathrm{Ge}$ & 2.550 & 25.421 & 18.902 & 20.500 & $0.028 \pm 0.037$ \\
$\mathrm{Sn}$ & 2.637 & 19.952 & 14.619 & 19.000 & $0.112 \pm 0.058$ \\
$\mathrm{Ga}$ & 2.426 & 27.331 & 20.737 & 20.300 & $-0.007 \pm 0.030$ \\
$\mathrm{Hg}$ & 2.835 & 12.233 & 8.653 & 13.800 & $0.251 \pm 0.090$ \\
$\mathrm{~Pb}$ & 2.962 & 11.943 & 8.247 & 11.400 & $0.147 \pm 0.054$ \\
$\mathrm{Ca}$ & 3.230 & 26.076 & 17.035 & 28.500 & $0.284 \pm 0.062$ \\
$\mathrm{Bi}$ & 3.124 & 11.275 & 7.535 & 13.080 & $0.322 \pm 0.059$ \\
$\mathrm{Ba}$ & 3.532 & 13.877 & 8.442 & 18.800 & $0.593 \pm 0.079$ \\
$\mathrm{Cd}$ & 2.552 & 19.894 & 14.787 & 18.000 & $0.078 \pm 0.053$ \\
\hline \multicolumn{5}{|c|}{}
\end{tabular}

The possibility of applying the BS scheme to at least the high electron density liquid metals, for which the RPA prescription is more reliable and the introduction of an ionic size in not mandatory to ensure the stability of the model system, has been checked on polyvalent metals. These systems turned out to be of simple experimental access $[5,8,9,17]$ and to sustain still detectable long living collective excitations. Surprisingly, a good estimate of $c_{\exp }$ was obtained within the BS scheme for some $s p$ liquid metals, namely $\mathrm{Hg}, \mathrm{Pb}, \mathrm{Sn}$ and $\mathrm{Ge}$, despite a greater ionic complexity was expected [13]. However, the discrepancies found for liquid $\mathrm{Ga}$, Al and $\mathrm{Bi}[9,10,17]$, which were 


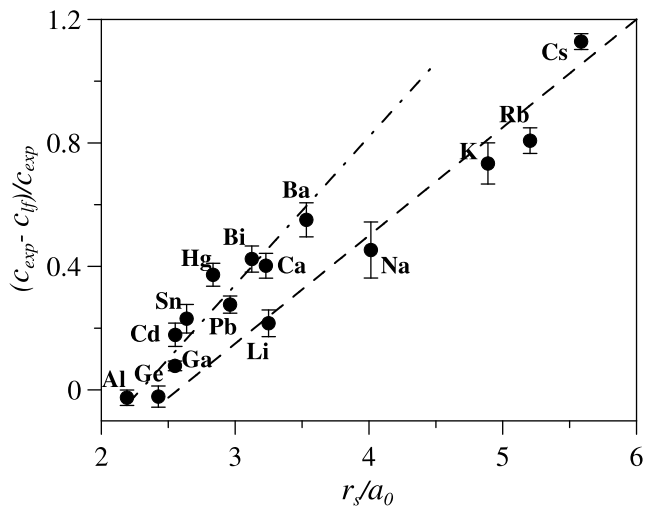

Figure 4. Relative deviation of the measured propagation velocity $c_{\exp }$ from the prediction with the exact screening wavevector, $c_{\mathrm{lf}}$ (see text), as a function of $r_{\mathrm{s}}$. The lines are guides for the eye.



Figure 5. Estimate for the ionic core parameter, $\left\langle R^{2}\right\rangle / 6+D_{\mathrm{p}}$, (see text), as a function of $r_{\mathrm{s}}$. The lines are guides for the eye.

the object of successive investigations, overhaul the good quantitative agreement observed in few cases and suggest that the agreement is fortuitous and arising from balancing effects between the approximated RPA dielectric function and the oversimplified ion-ion interaction. In figure 4 we show the relative deviation, as a function of $r_{\mathrm{s}}$, between the measured propagation velocity and its BS prescription, under both RPA and exact estimates of the screening wavevector, in alkali and $s p$-like metals available in literature. The results are also summarized in table I. The $r_{\mathrm{s}}$ values reported have been derived from the mean interstitial density of the corresponding fcc or bcc crystals, calculating the effective interstitial electron density. The BS model definitively fails in giving an overall prediction of the absolute values of the collective mode velocity even in the low $r_{\mathrm{s}}$ limit. [10,18]. The main conclusion of this study is that, although attractive, a simple application of the BS scheme to the alkali and $s p$-like liquid metals, even taking into account the electron correlations, does not produce reliable description of the ionic collective dynamics. The approximation of point-like ions should be left behind to provide a unified description. The effects of the finite size of the ion core on the low $Q$ limit of the collective mode frequency, still at high frequency, can be taken into account resorting to an interacting two-component model: a classical ion plasma in the strong coupling limit, with finite size of the ion core, and an interacting electron gas. Actually, the interacting parameter of the ionic plasma, derived from the mean Coulomb potential $U_{\text {int }}$, is, for liquid metals at melting temperature, far higher than $1(\sim 150)$, while the interacting parameter for the electron gas, derived from the correlation radius [19] $\left(0.543 r_{\mathrm{s}}\right)$, is roughly two orders of magnitude lower. Applying this model we can calculate the renormalized frequencies for the longitudinal mode throughout:

$$
\omega^{2}(Q)=\Omega_{\mathrm{p}}^{2}(Q)-\frac{1}{\left(4 \pi \mathrm{e}^{2}\right)} Q^{2} v^{2}(Q)\left[1-\frac{1}{\epsilon(Q)}\right],
$$

where $v(Q)$ is the effective ion-electron interaction potential [14]. Applying the low $Q$ approximations $\Omega_{\mathrm{p}}^{2}(Q) \sim \Omega_{\mathrm{p}}^{2}\left(1+D_{\mathrm{p}} Q^{2}\right)$ and $Q^{2} v^{2}(Q) /\left(4 \pi \mathrm{e}^{2}\right) \sim \Omega_{\mathrm{p}}^{2}\left[1-\left\langle R^{2}\right\rangle Q^{2} / 6\right]$, where $D_{\mathrm{p}}$ describes the low $Q$ plasma dispersion and $\left\langle R^{2}\right\rangle$ is the ion mean square radius, we obtain the following dispersion relation for the collective mode:

$$
\omega^{2}(Q) \cong Q^{2}\left(\frac{\Omega_{\mathrm{p}}}{k_{\mathrm{s}}}\right)^{2}+Q^{2} \Omega_{\mathrm{p}}^{2}\left[\frac{R^{2}}{6}+D_{\mathrm{p}}\right]+\mathcal{O}\left(Q^{4}\right) .
$$

The introduction of the ionic radius and the ion-electron interaction guarantees that, even when the dielectric function is not positive, $\omega^{2}(Q)$ can be positive-defined and hence a real-valued dispersion 
$\omega(Q)$ can exist. Applying this analysis to the experimental collective mode velocity data in alkali and $s p$-like metals [9] we can derive a reliable estimate for $\left\langle R^{2}\right\rangle / 6+D_{\mathrm{p}}$. The values obtained are also reported in table I and plotted against $r_{\mathrm{s}}$ in figure 5 .

The first observation one can draw from the results shown in figure 5 is that a rather simple trend is apparent although it is also evident that there is not an exact relationship between $\left\langle R^{2}\right\rangle / 6+D_{\mathrm{p}}$ and $r_{\mathrm{s}}$. In addition one can also observe that the parameter $\left\langle R^{2}\right\rangle / 6+D_{\mathrm{p}}$ becomes negative when the density of the system is increased. This is a quite interesting behaviour since, $\left\langle R^{2}\right\rangle / 6$ being positively defined, one can immediately guess that $D_{\mathrm{p}}$ can be negative. Actually, even if there is no experimental evidence of this effect coming from different sources, all the possible theories one can derive for a (classic) ion plasma [19] in the strong coupling limit provide a negative value for $D_{\mathrm{p}}$. The present observation is the first experimental indication of this behaviour which is also common to Bose and Fermi plasmas in the strong coupling limit [20]. A quantitative description of the dispersion term and of the electron gas contributions needs an improved derivation of the electron gas parameter and of the ion charge. These two quantities are related to each other in the present model and a formal derivation is rather difficult. At present, the only available suggestion stems from the observation that in the crystalline phase, where an accurate local density calculation of the electron states can be carried out, there exist a clear trend of the compressibility versus the interstitial electron density [21]. However, in a liquid metal even the concept of interstitial density should be considered with some care and the idea can be only taken as a starting point. For the time being, one can simply state that the proposed model is an accurate starting point and the details of the electron-ion potential have only a minor effect on the long wavelength, high frequency velocity, of the collective excitations.



Figure 6. Measured damping factors $\Gamma(Q) / \omega(Q)$, estimated for wavevectors $Q<Q_{0} / 2$, as a function of the atomic mass number.

Finally one can comment about the observed damping of the collective modes in liquid metals. Contrarily to what happens in crystals, where the phonon peak broadening is essentially ascribable to anharmonicity effects, which are strongly temperature dependent, the broadening of the density fluctuation peak in liquid metals is poorly sensitive to the temperature and is probably mainly a consequence of the disordered structure. Moreover, the strong tendency of the electron gas to screen fluctuations in the positive ion density means that the electrons will closely follow the system compression waves trying to compensate the local charge displacement. This derives from an additional contribution to the sound waves attenuation from the electron gas screening. A simple theoretical approach for the processes giving rise to the mode damping is not available. The only straightforward interaction one can evaluate is the electron-phonon interaction [22] which is largely too small to produce observable effects. In general, the experimental finding is that the damping is an almost linear function of the momentum transfer in the mesoscopic range, i.e. for wavevectors between $\sim 0.1 Q_{0}$ and $\sim 0.5 Q_{0}$, so that the ratio $\Gamma(Q) / \omega(Q)$ is practically constant. Quite a large damping is present in all systems, as reported in figure 6 , where the systems are seen to be grouped into categories with seemingly no relationship existing with the electron density. Nonetheless one can observe that in the case of liquid Ga no temperature dependence of the damping is present in going from $320 \mathrm{~K}$ just above the melting point up to $970 \mathrm{~K}[9]$. This behaviour was attributed to the electron interactions as the source of the damping in Ga since on increasing the temperature one would expect a strong increase of the damping if it is to be attributed mainly to ion dynamics. On the contrary, the electron interactions can be safely assumed to be temperature independent since the thermal energy is much smaller than the Fermi energy. The only system which behaves in a different way is liquid Bismuth, whose electronic structure is thought to be peculiar. Indeed this system shows a very high 
damping, with a different $Q$-trend [10], according to its high electrical resistivity. Therefore, the important conclusion one can draw is that further experimental investigations should be performed in order to understand the role of the various contributions to the damping processes which are present in liquid metals.

\section{References}

1. Copley J.R.D., Rowe J.M., Phys. Rev. Lett., 1974, 32, 49.

2. Bodensteiner T., Morkel C., Gläser W., Dorner B., Phys. Rev. A, 1992 45, 5709; Verkerk P., de Jong P. H. K., Arai M., Bennington S.M., Howells W.S., Taylor A.D., Physica B, 1992, 180-181, 834; Bermejo F.J., et al., Phys. Rev. Lett., 2000, 85, 106; Cabrillo C., et al., Phys. Rev. Lett., 2002, 89, 075508-1; Bermejo F.J., et al., Phys. Rev. B, 2005, 72, 1041103; Ruiz-Martin M.D., et al., Phys. Rev. B, 2007, 75, 224202.

3. Bove L.E., et al., Phys. Rev. Lett., 2000, 85, 5352.

4. Bove L.E., et al., Phys. Rev. B, 2003, 68, 024208.

5. Sinn H., et al., Phys. Rev. Lett., 1997, 78, 1715; Pilgrim W.C., et al., J. Non-Cryst. Solid., 1999, 250-52, 96a; Scopigno T., et al., Phys. Rev. Lett., 2000, 85, 4076; Scopigno T., Ruocco G., Sette F, Rev. Mod. Phys., 2005, 77, 881a; Hosokawa S., et al., Phys. Rev. B, 2001, 63, 134205; Hosokawaa S., et al., Chem. Phys., 007 292, 253.

6. Bell H.G., Phys. Lett. A, 1973, 45, 479a; Bafile U., et al., Phys. Rev. Lett., 1990, 6, 2394a; Cunsolo A., et al., Phys. Rev. Lett., 1998, 80, 3515.

7. Friedel J., Philos. Mag. B, 1953, 43, 153.

8. Bove L.E., et al., Phys. Rev. Lett., 2001, 87, 215504.

9. Bove L.E., et al., Phys. Rev. B, 2005, 71, 014207.

10. Sani L., et al., J. Non-Cryst. Solid., 2007, 353, 3139.a

11. Pines D., Nozieres Ph. The Theory of Quantum Liquids. W. A. Benjamin Inc., New York, 1966.

12. Blairs S., J. of Coll. Int. Sci, 2006, 302, 312.

13. Bove L.E., Philos. Mag. B, 2002, 82, 265a; Bove L.E., J. Non-Cryst. Solid., 2002, 307, 842.

14. Sacchetti F., et al., Phys. Rev. B, 2003, 67, 014207.

15. Iwamoto N., Phys. Rev. A, 1984, 30, 3289.

16. Gori-Giorgi P., Sacchetti F., Bachelet G.B., Phys. Rev. B, 2000, 61, 7353 and references therein.

17. Scopigno T., et al., Phys. Rev. E, 2001, 63, 011210

18. Papacostantopoulos D.A. Handbook of the Band Structure of Elemental Solids. Plenum, New York, 1986.

19. Ichimaru S.Rev. Mod. Phys., 1982, 54, 1017.

20. Böhm H.M., Conti S., Tosi M.P., J. Phys.: Condens. Matter, 1996, 8, 781.

21. Moruzzi V.L., Janak J.F., Williams A.R., Calculated Electronics Properties of Metals. Pergamon, New York, 1978.

22. March N.H., Liquid Metals. Cambridge University Press, Cambridge, 1990. 


\title{
Флуктуації іонної густини в рідких металах: сильно взаємодіюча іон-електронна плазма
}

\author{
Л.Е.Бове ${ }^{1}$, К.Петрілло을 Ф.Саккетті²
}

1 Відділ фізики густих рідин Національної Ради Досліджень, Університет Парижу, Париж, Франція

2 Фізичний факультет, Університет Перуджі, Перуджя, Італія та Національна Рада Досліджень, Центр SOFT, Рим, Італія

Отримано 20 листопада 2007 р.

Робиться спроба узагальненого опису динаміки рідких металів на основі взаємодіючої двокомпонентної моделі для іон-електронної плазми. Швидкість поширення колективних мод у лужних та полівалентних металах, отримана з експериментів по непружному розсіянню нейтронів та рентгенівських променів, порівнюється з оцінками, отриманими різними теоретичними наближеннями для сильно взаємодіючої плазми. Використовуючи точні результати для кореляційної енергії електронного газу та враховуючи ефекти скінченого розміру іонів, отримано в цілому добрий опис великого набору рідких металів. Спостережувана тенденція до згасання колективних мод у рідких металах також обговорюється в рамках двокомпонентного опису.

Ключові слова: рідкі метали, динаміка, непружне розсіяння нейтронів та рентгенівських променів, електронна густина

PACS: $61.12 .-q, 61.25 . \mathrm{Mv}, 71.10 . \mathrm{Ca}$ 